\title{
Avaliação das ações de controle da leishmaniose visceral (calazar) em uma área endêmica do Estado da Bahia, Brasil (1995-2000)
}

\author{
Evaluation of control measures for visceral \\ leishmaniasis (kala azar) in an endemic area \\ in Bahia, Brazil (1995-2000)
}

Simone Souza de Oliveira 1,2

Tânia Maria de Araújo 3

\footnotetext{
1 Fundação Nacional de Saúde - Pólo Base de Feira de Santana. Rua Saturno $s / n$, Feira de Santana, BA 44100-000, Brasil. simones.o@ig.com.br 2 Departamento de Ciências Biológicas, Universidade Estadual de Feira de Santana. BR-116, $\mathrm{Km} \mathrm{3}$, Feira de Santana, BA 44031-460, Brasil.

3 Departamento de Saúde, Universidade Estadual de Feira de Santana. BR-116, $\mathrm{Km}$ 3, Feira de Santana, BA 44031-460, Brasil.
}

Abstract This study evaluated the Control Program for Visceral Leishmaniasis in the city of Feira de Santana, Bahia, Brazil. Information was collected on control measures from 1995 to June 2000, and focused on periodicity, coverage, and incidence trends in the target areas. The dependent variable was the incidence rate for human cases, and independent variables were: percentage of buildings visited in the canine survey and buildings sprayed, number of cycles in the canine survey and spraying, and canine prevalence rate. To evaluate the correlation between control measures and the human visceral leishmaniasis incidence rate, we calculated the Pearson's correlations coefficients and performed linear regression analysis. No association was observed between the prevalence of dogs with positive serology and the incidence rate of human cases. The variables that remained in the final model and that proved most effective in controlling the disease were: percentage of buildings visited during the survey and spraying and number of cycles in the canine survey, capable of explaining 40\% of the incidence of human cases. The findings constitute relevant material for the discussion of effectiveness in control measures for visceral leishmaniasis.

Key words Visceral Leishmaniasis; Vector Control; Program Evaluation

Resumo Este estudo objetivou avaliar as ações do Programa de Controle da Leishmaniose Visceral no Município de Feira de Santana, Bahia, Brasil. Foram coletadas informações das ações no período de 1995 a junho de 2000, considerando-se a periodicidade e cobertura, e a evolução da incidência nas áreas trabalhadas. A variável dependente foi a incidência de casos humanos $e$ as variáveis independentes foram: percentual de prédios trabalhados para inquérito canino $e$ prédios borrifados, número de ciclos de inquérito canino e borrifação e prevalência canina. Para avaliar a correlação entre as ações e a incidência de casos humanos calculou-se os coeficientes de correlação de Pearson e procedeu-se à analise de regressão linear. Não foi observada associação entre a prevalência de cães com sorologia positiva e a incidência de casos humanos. As variáveis que permaneceram no modelo final e que tiveram efetividade mais destacada no controle da doença foram: percentual de prédios trabalhados para inquérito e borrifação e número de ciclos de inquérito canino, capazes de explicar 40\% da incidência de casos humanos. Os achados constituem material relevante para discussão da efetividade das ações de controle da leishmaniose visceral.

Palavras-chave Leishmaniose Visceral; Controle de Vetores; Avaliação de Programas 
Devido à importância de se avaliar as ações desenvolvidas no controle das doenças endêmicas, principalmente quanto à sua real efetividade, tendo em vista as condições como estas são desenvolvidas, analisou-se as ações do Programa de Controle da Leishmaniose Visceral ou calazar no Município de Feira de Santana, Bahia, Brasil, desde o início de sua implantação.

Historicamente, a campanha contra a leishmaniose visceral (CCL) foi criada em 1953, com o objetivo de estudar e combater a leishmaniose visceral (LV), tendo em vista o aumento do número de casos da doença no país (Silva, 1957).

Durante os anos 60, a CCL foi interrompida, sendo novamente retomada em 1980, quando detectou-se um grande incremento na prevalência da doença. Nesse período, a Bahia destacou-se entre os focos endêmicos mais importantes do país (Lacerda, 1994).

Somente em 1985, o Programa de Controle da Leishmaniose (PCL) foi implantado na Bahia com muitas limitações, em virtude do pouco conhecimento da endemia, da insuficiência de recursos humanos e da carência de recursos materiais (MS, 1985).

As atividades desenvolvidas consistiam em busca ativa, educação sanitária e divulgação, além de borrifação focal, com a aplicação de inseticida nos domicílios com casos humanos ou cães suspeitos. O inquérito canino não era realizado por falta de laboratório de apoio diagnóstico (MS, 1985).

A partir de 1986, o PCL tomou impulso com o incremento das atividades de vigilância entomológica e aplicação de inseticidas de poder residual nas paredes internas e externas do domicílio; identificação de cães com sorologia positiva e sua posterior eliminação; detecção ativa e passiva dos casos humanos suspeitos com a garantia do diagnóstico e tratamento dos doentes, tendo em vista a implantação da reação de Imunofluorescência Indireta (IFI), técnica sorológica utilizada para evidenciar a infecção entre cães e humanos, visando assim, os três elementos da cadeia de transmissão da doença: o vetor Lutzomyia longipalpis; o principal reservatório, cão doméstico, e o homem (MS, 1996).

No período de 1985 a 1998, o Estado da Bahia passou a ser responsável pela notificação do maior número de casos humanos de LV do Brasil, excetuando-se os anos de 1993 e 1994, quando foi ultrapassado pelo Estado do Piauí (MS, 1999).

A distribuição geográfica da doença na Bahia é ampla, com maior concentração na região central do estado. Na última década, veri- ficou-se expansão para o oeste, norte e nordeste, atingindo regiões litorâneas, representando um padrão diverso do classicamente descrito. Em anos recentes, outras áreas foram atingidas, indicando expansão para o sul do estado, tradicionalmente indene para a doença (SESAB, 1999).

Em 1995, ocorreu uma epidemia de LV no Município de Feira de Santana. Vale ressaltar que, no período entre 1985 a 1993 foram notificados 36 casos de LV como procedentes do Feira de Santana, provavelmente pelo fato do município ser referência para a microrregião, recebendo encaminhamento de pacientes para diagnóstico e tratamento no Hospital Geral Clériston Andrade (HGCA), onde os casos foram registrados, porém não investigados quando à sua procedência.

Em 1994, 14 casos de LV foram notificados e após investigação constatou-se que dez eram procedentes do Município de Feira de Santana, o que caracterizou o início da epidemia. Este número elevou-se para cem casos em 1995, mantendo-se elevado em 1996, diminuindo nos anos seguintes (Figura 1).

Após a constatação da epidemia, o PCL foi implantado no Município de Feira de Santana em 1995, e a avaliação de suas ações no período de 1995 a 2000 constitui o objetivo do presente estudo, tomando como base o efeito das ações desenvolvidas na redução da incidência da doença.

\section{Material e métodos}

O Município de Feira de Santana situa-se a leste do Estado da Bahia, a 107Km da capital, entre a zona da mata e o sertão, numa área de transição denominada agreste baiano. Cerca de $96 \%$ da área do município está inserida no “polígono das secas” (Governo do Estado da Bahia, 1998).

Pela importância de sua localização geoeconômica, entre o litoral e o interior, Feira de Santana é um dos maiores entroncamentos rodoviários do interior do país, cortado por três rodovias federais: BR 101, BR 116 e BR 324, e quatro rodovias estaduais: BA 052, BA 502, BA 503 e BA 504, favorecendo intenso fluxo de população, mercadoria e dinheiro, num entreposto que liga o Nordeste ao Centro-Sul, a fronteira da capital Salvador com o sertão (Governo do Estado da Bahia, 1998).

A área do município é de $1.338,1 \mathrm{Km}^{2}$. Predomina clima seco a subúmido e semi-árido, a temperatura média anual é de $23,5^{\circ} \mathrm{C}$ e média mínima $19,6^{\circ} \mathrm{C}$. As chuvas de inverno normal- 
Número de casos humanos de leishmaniose visceral no Município de Feira de Santana, Bahia, no período de 1985 a junho de 2000.

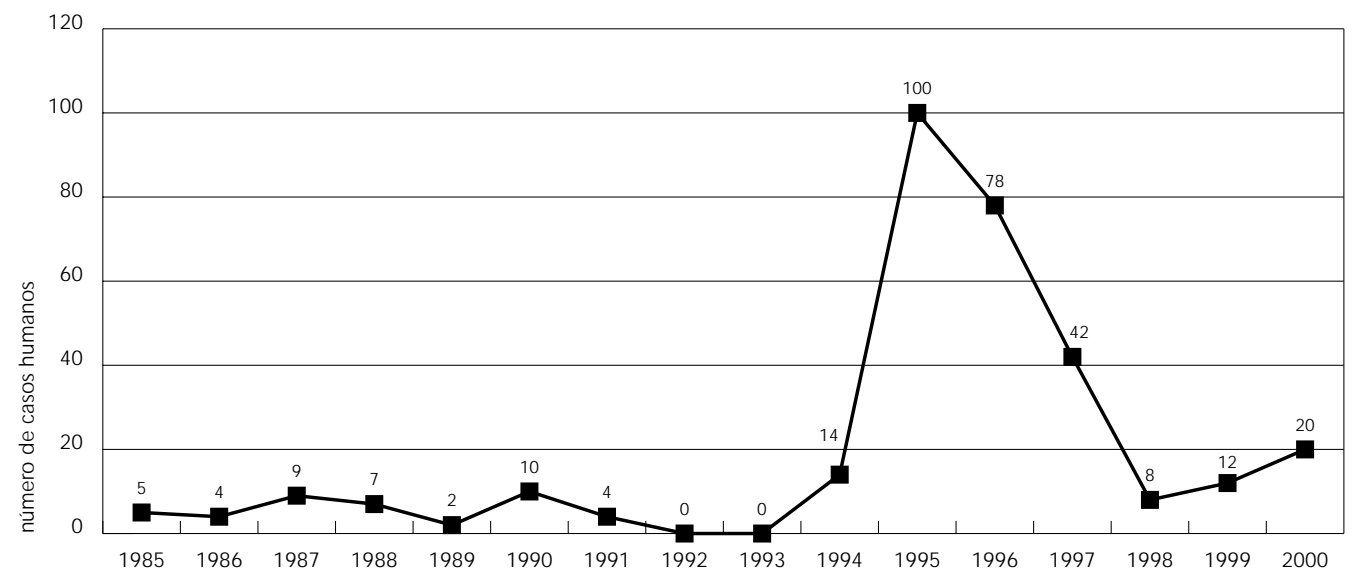

mente ocorrem de maio a agosto. Apresenta pluviosidade média anual máxima de $1.595 \mathrm{~mm}$ e mínima de $444 \mathrm{~mm}$ (Governo do Estado da Bahia, 1998).

Feira de Santana ocupa a segunda posição entre os municípios mais populosos do estado, com um total de 498.638 habitantes (IBGE, 2000).

Este município possui 660 localidades, 90 na zona urbana e 570 na zona rural, de acordo com o sistema de localidades (SISLOC) adotado pela Fundação Nacional de Saúde (FUNASA/MS). A área do estudo foi constituída por 124 localidades onde foram desenvolvidas as atividades do PCL, sendo trinta bairros localizados na zona urbana e 58 localidades rurais (sítios, fazendas e vilas) que registraram casos humanos, além de 36 localidades circunvizinhas àquelas com casos humanos, no período de 1995 a junho de 2000.

A população foi composta por todos os casos humanos notificados no período, obtidos após levantamento de todas as fontes notificadoras: boletim da FUNASA CCL 22 - Registro do Atendimento de Casos Humanos de Leishmaniose Visceral e Tegumentar, e boletim de notificação semanal de doenças transmissíveis da Secretaria Estadual de Saúde (SESAB), por localidade e ano de ocorrência.

De acordo com a metodologia do programa, as atividades de inquérito canino e borrifação residual com inseticida piretróide devem ser realizadas nas localidades com registro de casos humanos, positividade canina e presença do vetor, em ciclos semestrais (dois ciclos anuais), obedecendo raio de um quilômetro a partir do caso humano na zona rural e $200 \mathrm{~m}$ na zona rural, sendo progressivamente ampliado pela incorporação de áreas adjacentes, na medida em que forem sendo identificados cães positivos.

O inquérito canino consistiu na coleta de sangue da orelha de $100 \%$ dos cães em papel de filtro. As amostras foram examinadas pela técnica de IFI no Laboratório de Sorologia da Universidade Estadual de Feira de Santana (UEFS) e Laboratório de Saúde Pública da FUNASA. Os cães soropositivos foram eliminados.

A borrifação com inseticida piretróide atingiu as paredes internas e externas do domicílio e anexos (galinheiros, estábulos, depósitos).

Os dados do PCL foram obtidos dos boletins da FUNASA PCL-1A - Operação de Controle do Reservatório Canino e PCL-3A - Operação de Inseticida - Borrifação, onde foram coletadas as seguintes informações por localidade: número de prédios existentes, número de prédios trabalhados, de prédios trabalhados para inquérito canino, de ciclos de inquérito canino, de amostras caninas coletadas, de amostras caninas positivas, de prédios borrifados e número de ciclos de borrifação por ano trabalhado.

Não foram utilizadas as informações resultantes do inquérito entomológico por ter sido realizado de forma descontinuada, abrangendo pequeno número de localidades e domicí- 
lios, e das atividades educativas e de mobilização comunitária, também sem registro contínuo, o que impossibilitou sua quantificação.

Para avaliar as medidas do PCL, tomou-se como base o efeito das ações desenvolvidas no município, ou seja, a efetividade, considerando que efetividade é o grau em que determinada intervenção, procedimento, regime ou serviço produz um resultado benéfico, quando empregado no "mundo real”, em uma população definida; é o resultado verdadeiramente observado nas condições habituais de uso (Pereira, 1995).

A seleção das ações avaliadas foi baseada na disponibilidade das informações que apresentaram maior consistência e sistematização no período. Dentre as principais ações do PCL foram consideradas as variáveis independentes, aquelas relacionadas às ações desenvolvidas para o controle do vetor e reservatório doméstico.

Com relação ao reservatório canino foram investigados: percentual de prédios trabalhados para inquérito canino, número de ciclos anuais de inquérito canino e prevalência canina. Em relação à operação inseticida foram: percentual de prédios borrifados e número de ciclos anuais de borrifação.

A variável dependente foi a incidência de casos de LV por 10 mil habitantes por localidade urbana e rural do Município de Feira de Santana.

Inicialmente, foram calculadas as incidências de LV nas áreas estudadas e avaliadas as ações implementadas nessas áreas, enfatizando-se o percentual de cobertura e a periodicidade.

$\mathrm{Na}$ análise da efetividade das ações do PCL e a distribuição da incidência da LV, no período estudado, realizou-se teste de correlação de Pearson entre a incidência de casos humanos de LV no município, as ações do PCL e a análise de regressão linear considerando cada uma das ações do PCL.

O coeficiente de regressão múltipla expressa a intensidade de correlação entre uma variável dependente, a incidência de casos humanos de LV com as demais variáveis independentes. O uso desse método pode explicar a variação de uma variável dependente na presença de uma variável independente (regressão linear simples), ou explicar a variação de uma variável dependente na presença de um conjunto de variáveis independentes.

A análise de regressão linear múltipla foi desenvolvida nas seguintes etapas: pré-seleção das variáveis (análise bivariada) e regressão linear múltipla, com procedimento "de trás para frente” para seleção das variáveis do modelo final.
Para pré-seleção das variáveis, avaliou-se a associação entre a variável dependente e cada uma das variáveis independentes (ações do PCL), observando-se, na análise de regressão linear simples, um $\mathrm{p} \leq 0,25$. Na realização da regressão múltipla, com as variáveis que atenderam ao critério anterior de entrada para manutenção da variável no modelo, considerou-se um $\mathrm{p} \leq 0,17$. Variáveis com um valor de $\mathrm{p}$ acima de 0,17 no teste foram sendo retiradas a cada etapa, resultando um modelo de avaliação da efetividade das ações do PCL no controle da doença.

Um aspecto crucial para a regressão é a escolha do valor de $\alpha$ para o julgamento da importância das variáveis. Estudos avaliando níveis de escolha em regressão linear e análise discriminante têm mostrado que a escolha de p igual a 0,05 é muito conservadora e, muitas vezes, têm excluído variáveis importantes. Assim, a escolha de "um nivel $\alpha$ que varie entre 0,15 a 0,20 tem sido fortemente recomendado" (Hosmer \& Lemeshow, 1989:108).

As análises foram realizadas tomando-se por base as ações desenvolvidas num ano em relação a incidência da doença no ano seguinte, ou seja, as atividades desenvolvidas em 1995 e a incidência observada em 1996 e assim sucessivamente, tendo como resultado final a incidência de LV no ano 2000.

Para análise das variáveis foi utilizado o programa estatístico SPSS versão 8.0.

\section{Resultados}

A LV apresentou coeficiente de incidência de 22,8/100 mil habitantes no ano de 1995 (pico da epidemia), diminuindo nos anos seguintes. A distribuição anual do número de casos humanos por local de ocorrência foi mais elevada na zona rural em todo período do estudo. A prevalência canina foi elevada em 1995, início da epidemia, inclusive na zona urbana, e em 1997, mantendo-se em níveis mais baixos nos demais anos do estudo (Tabela 1).

No ano de 1995, as atividades desenvolvidas concentraram-se na zona urbana do município, onde foram notificados os primeiros casos da doença. Com o início das ações, a prevalência canina e a incidência de casos humanos apresentaram redução em cerca de $50 \%$ e as atividades de inquérito canino e borrifação foram realizadas na sua maioria em dois ciclos. Em 1996, houve uma redução das ações sendo intensificadas em 1997, principalmente quanto à sua periodicidade. Nos anos seguintes, as atividades do PCL foram reduzidas prin- 
Coeficiente de incidência de casos humanos e prevalência canina global de leishmaniose visceral por zona de ocorrência no Município de Feira de Santana, Bahia, no período de 1995 a junho de 2000.

\begin{tabular}{|c|c|c|c|c|c|c|}
\hline \multirow[t]{2}{*}{ Ano } & \multicolumn{3}{|c|}{$\begin{array}{l}\text { Coeficiente de incidênica/ } \\
100 \text { mil habitantes }\end{array}$} & \multicolumn{3}{|c|}{ Prevalência canina (\%) } \\
\hline & Global & Zona urbana & Zona rural & Global & Zona urbana & Zona rural \\
\hline 1995 & 22,8 & 20,7 & 36,9 & 1,6 & 1,7 & 1,1 \\
\hline 1996 & 17,3 & 8,4 & 79,6 & 0,8 & 0,5 & 1,8 \\
\hline 1997 & 9,1 & 4,2 & 43,2 & 2,1 & 1,7 & 5,6 \\
\hline 1998 & 1,7 & 1,5 & 3,4 & 0,7 & 0,6 & 3,3 \\
\hline 1999 & 2,5 & 1,2 & 11,6 & 1,0 & 0,5 & 4,0 \\
\hline 2000 & 4,0 & 2,1 & 19,0 & 0,6 & 0,3 & 2,1 \\
\hline
\end{tabular}

cipalmente quanto ao número de ciclos de trabalho, observando-se uma tendência na elevação dos casos humanos no início do ano 2000 (Figura 2).

Na zona rural, no ano de 1995, o coeficiente de incidência de casos humanos foi de 36,9/100 mil habitantes e a prevalência canina de $1,13 \%$. As atividades de inquérito canino e borrifação foram realizadas em apenas um ciclo de cobertura anual. A incidência da doença elevou-se para 79,6/100 mil habitantes. No período de 1996 a 1997, houve aumento do percentual de prédios trabalhados para inquérito canino e borrifação, e uma redução dos casos humanos; porém, a prevalência canina elevou-se no mesmo período. A partir de 1998 o percentual de prédios trabalhados para inquérito canino diminuiu, a incidência da doença aumentou consideravelmente, tanto quanto a prevalência canina, com tendência a continuar aumentando no ano 2000 (Figura 3).

O teste de correlação de Pearson mostrou que a associação entre a prevalência canina e a incidência de casos humanos foi fraca $(0,1180)$ e não apresentou significância estatística ( $\mathrm{p}=$ 0,369 ), tomando como base a prevalência canina em um ano e a incidência de casos humanos no ano seguinte por localidade para o período estudado (Tabela 2 ).

Em relação a outras variáveis, a análise de correlação mostrou associação positiva entre a incidência de casos humanos e o percentual de prédios borrifados e o de prédios trabalhados para inquérito canino, isto significa que estas variáveis variaram na mesma direção, sem que isto implique relação de causa e efeito (Tabela 2).

Considerando a incidência de casos humanos e as variáveis número de ciclos de borrifação e de ciclos de inquérito canino, a associação foi negativa, ou seja, o aumento da variável independente leva à diminuição da variável dependente (Tabela 2).

As variáveis percentual de prédios borrifados, números de ciclos de inquérito canino e percentual de prédios de inquérito canino estavam correlacionadas à incidência de LV, em níveis estatisticamente significantes, ainda que as correlações observadas tenham sido medianas (Tabela 2).

De acordo com os parâmetros estabelecidos para os procedimentos da análise de regressão linear múltipla, obteve-se o modelo final. Nesse modelo permaneceram as variáveis percentual de prédios trabalhados para inquérito canino, percentual de prédios borrifados e números de ciclos de inquérito canino. Observou-se associação positiva entre percentual de prédios trabalhados para inquérito canino e de prédios borrifados, enquanto que para número de ciclos de inquérito canino e incidência de LV observou-se associação negativa (Tabela 3 ).

A intensidade de correlação entre o conjunto de variáveis independentes e a incidência de casos humanos indicou associação forte ( $\mathrm{r}=$ 0,63876), porém, a variação da incidência que foi explicada pelo conjunto das variáveis independentes incluídas no modelo de regressão linear múltipla foi de $40 \%$ (Tabela 3 ).

\section{Discussão}

Para analisar as ações do PCL deve-se levar em consideração que a área trabalhada foi delimitada de acordo com a ocorrência de casos humanos da doença, ampliada à medida que novas localidades com cães positivos e/ou presença do vetor foram sendo identificadas.

A borrifação com inseticida piretróide foi realizada em menor intensidade, principal- 
Figura 2

Distribuição da incidência de casos de leishmaniose visceral de acordo com a prevalência canina, percentual de prédios trabalhados no inquérito canino e borrifação na zona urbana do Município de Feira de Santana, Bahia, no período de 1995 a junho de 2000.

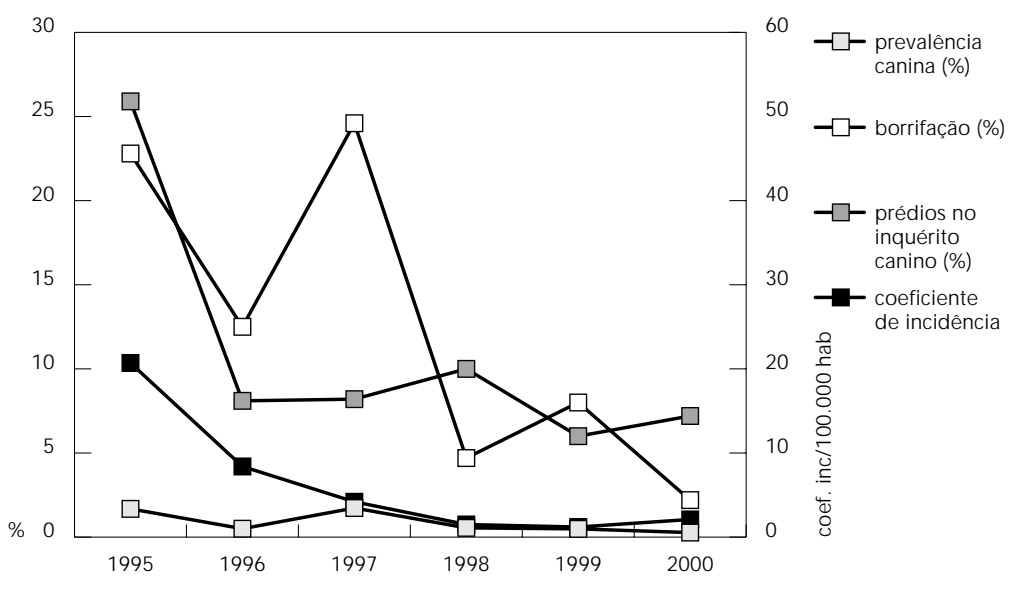

Figura 3

Distribuição da incidência de casos de leishmaniose visceral de acordo com a prevalência canina, percentual de prédios trabalhados no inquérito canino e borrifação na zona rural do Município de Feira de Santana, Bahia, no período de 1995 a junho de 2000.

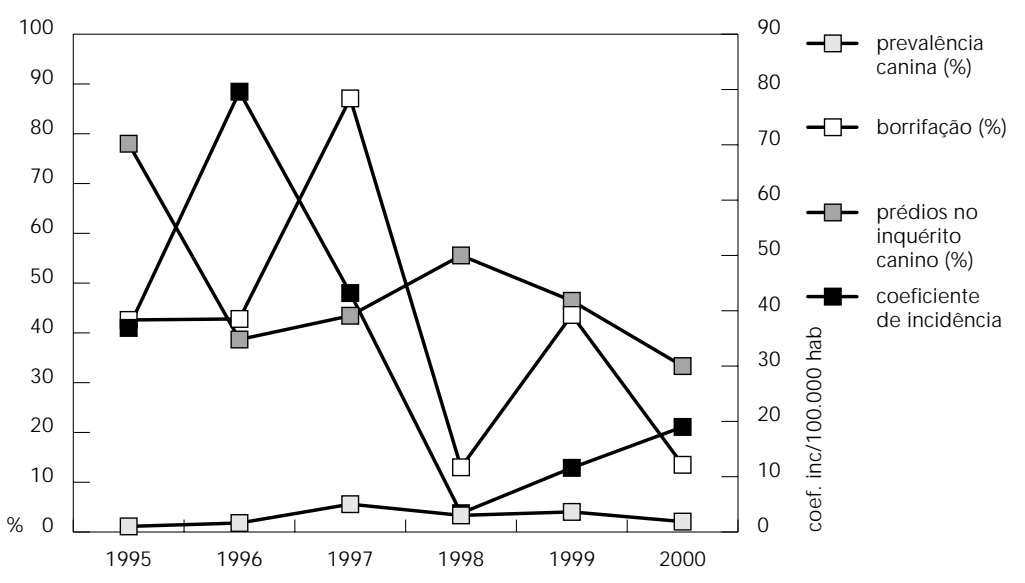

mente devido a falta de recursos humanos e materiais ao longo do período estudado.

Com base na analise das ações do PCL, ano a ano, observou-se que sua implantação no município não obedeceu as recomendações do PCL. Assim, as limitações colocadas para avaliação das mesmas são diversas e de diferentes ordens. Dentre as limitações observadas podese destacar que as ações do PCL não foram implementadas de forma sistemática e contínua no período, ocorrendo variações nas áreas trabalhadas e no percentual de cobertura de cada ação por zona de ocorrência; a qualidade das informações disponíveis sobre o desenvolvimento das ações variaram e sofreram influência de disponibilidade de recursos materiais e humanos; a implementação das ações dependeu diretamente da ocorrência de casos humanos nas áreas estudadas, portanto, a incidência da doença determinava a intensidade e a regularidade das ações adotadas. Logo, o uso dos métodos epidemiológicos somente pode ser usado por aproximação (uma vez que o uso clássico desses métodos sustenta-se no pressuposto de que o efeito variaria em função da exposição).

Mesmo considerando as limitações para a realização de procedimentos analíticos para avaliação das ações do PCL em Feira de Santana, optou-se aqui por fazê-lo devido à necessidade de se avaliar, mais detalhadamente o desempenho das ações do PCL, principalmente dentro da realidade operacional da maioria dos municípios brasileiros e a possibilidade de se propiciar informações com nível de análise mais aprofundado e se obter estimativas mais consistentes, de modo a permitir repensar e fortalecer se determinadas ações foram as razões que justificaram a realização destes procedimentos. No entanto, ressalta-se que os achados produzidos, a partir do uso destes procedimentos, devem ser avaliados com cautela.

$\mathrm{Na}$ área do estudo foram detectados cães com características clínicas da doença, tais como, alongamento das unhas, emagrecimento, queda de pêlos e feridas no focinho e orelhas, sugestivas de LV; porém, nos anos de 1995 e 1996, dos cães com sorologia positiva, 76,8\% e $89,5 \%$, respectivamente, eram cães aparentemente sadios, ou seja, o que reforça os achados da literatura, da importância do inquérito canino na identificação de cães infectados (Oliveira et al., 1998).

Senra et al. (1986) já detectaram em Santarém, Pará, a importância epidemiológica de cães clinicamente suspeitos e aparentemente sadios no índice de positividade de cães em áreas de transmissão da doença. No Rio de Ja- 
Coeficiente de correlação de Pearson, considerando-se a incidência de casos humanos de leishmaniose visceral e as ações do Programa de Controle da Leishmaniose no Município de Feira de Santana, Bahia, no período de 1995 a junho de 2000.

\begin{tabular}{|c|c|c|c|c|c|}
\hline $\begin{array}{l}\text { Incidência de casos } \\
\text { humanos de leishmaniose } \\
\text { visceral/10.000 habitantes }\end{array}$ & $\begin{array}{l}\text { Ciclos de } \\
\text { borrifação }\end{array}$ & $\begin{array}{l}\text { Prédios } \\
\text { borrifados (\%) }\end{array}$ & $\begin{array}{l}\text { Ciclos } \\
\text { inquérito } \\
\text { canino }\end{array}$ & $\begin{array}{l}\text { Prédios } \\
\text { inquérito } \\
\text { canino (\%) }\end{array}$ & $\begin{array}{l}\text { Prevalência } \\
\text { canina }\end{array}$ \\
\hline & $-0,2368$ & 0,4910 & $-0,3385$ & 0,4580 & 0,1180 \\
\hline & $p=0,117$ & $p=0,001$ & $p=0,008$ & $p=0,000$ & $p=0,369$ \\
\hline
\end{tabular}

Tabela 3

Modelo final obtido na análise de regressão linear múltipla, tendo a incidência de casos humanos por 10 mil habitantes como variável dependente e as demais ações do Programa de Controle da Leishmaniose como variável independente no Município de Feira de Santana, Bahia, no período de 1995 a junho de 2000.

\begin{tabular}{lrrr}
\hline Ações do programa de controle da leishmaniose & $\beta$ & Erro padrão de $\beta$ & Valor de $p$ \\
\hline Prédios de inquérito canino (\%) & 1,207129 & 0,519040 & 0,0252 \\
Prédios borrifados (\%) & 0,981432 & 0,376646 & 0,0128 \\
Ciclos de inquérito canino (n) & $-57,583997$ & 23,237873 & 0,0175 \\
\hline
\end{tabular}

$R=0,63876 ; R^{2}=0,40802$.

neiro, Marzochi (1985) constatou que $63,2 \%$ dos cães infectados não apresentavam sinais clínicos da doença.

Para Marzochi et al. (1986) e Costa et al. (1990), a introdução de cães doentes em determinadas áreas pode estar associada à migração, uma vez que estes animais, muitas vezes, são trazidos infectados da região de origem. As famílias ao se instalarem em áreas de poucos recursos, de desmatamento recente, em zonas peri-urbanas, onde já existem os flebotomíneos, contribuem para o aparecimento da doença em área urbana. Contudo, outros reservatórios selvagens primários podem simultaneamente existir, e isto explicaria alguns aspectos obscuros da cadeia natural desta doença (Sherlock, 1996, 1997).

O Didelphis albiventris foi o primeiro mamífero não canídeo a ser encontrado naturalmente infectado com L chagasi no continente americano. Com relação à presença de raposas na área estudada, $47,2 \%$ da população pesquisada afirmou tê-las visto próximo ao domicílio, principalmente na zona rural (Sherlock, 1996; Sherlock et al., 1984).

Analisando os coeficientes de Correlação de Pearson, verificou-se que a correlação entre a prevalência canina e a incidência de casos humanos no município foi fraca, não tendo sido estatisticamente significante. $\mathrm{Na}$ análise de regressão linear múltipla, resultado similar foi também observado, a prevalência canina não permaneceu no modelo final de análise.

A fraca associação entre a prevalência canina e a incidência de casos humanos pode estar relacionada a diversos fatores: baixa sensibilidade do teste de IFI no eluato de sangue, já demonstrado por Paranhos-Silva et al. (1996) em Jequié, quando comparou os testes de ELISA e IFI para infecção por Leishmania em soro de cães, encontrando $98 \%$ de sensibilidade quando utilizou-se a reação de ELISA e 78\% para IFI. Resultados semelhantes ao encontrado por Evans et al. (1990), que testou 405 amostras (soro e eluato) de cães, cujos resultados confirmaram maior sensibilidade da reação de ELISA em relação à de IFI no soro e menor sensibilidade do eluato, com $38 \%, 17 \%$ e $8 \%$, respectivamente.

Em estudo de intervenção controlado, realizado por Dietze et al. (1997) no Espírito Santo, com objetivo de avaliar o papel de cães infectados na transmissão da doença para o homem, verificou-se que, após a eliminação de 
cães soro positivos na área de intervenção, a soropositividade humana aumentou na mesma proporção que na área onde os cães soropositivo não foram eliminados. O papel do homem como um significante reservatório para LV é proposto para explicar os resultados deste estudo.

Ashford et al. (1998), em Jacobina, Bahia, realizou estudo similar em cães e observou que a soropositividade diminuiu na área de intervenção, por outro lado, a prevalência na área controle não mudou significativamente no período do estudo.

A observação da curva de casos humanos e dos índices da sorologia em cães no país revela que na última década foi constatado aumento dos casos humanos apesar da redução das taxas de soropositividade canina (Vieira \& Coelho, 1998).

Com base nos procedimentos analíticos observou-se que o percentual de prédios de inquérito canino e o de prédios borrifados apresentaram associação positiva, tendo em vista que, o PCL é implementado à medida que a incidência da doença aumenta, conseqüentemente, quanto maior o número de casos humanos maior será o percentual de cobertura para inquérito canino e borrifação.

Quanto ao número de ciclos de inquérito canino, a associação foi negativa, isto sugere que, a realização das ações em dois ciclos anuais se mostraram mais efetivas no controle da doença.

Os achados do presente estudo devem ser avaliados com cautela, uma vez que, o período do estudo é relativamente pequeno para se medir a efetividade das ações, porque estas só são realizadas após o aparecimento dos casos humanos. Ao longo do período estudado surgiram novas localidades com registro de casos humanos, que foram incorporados à análise, $o$ que implicou a diminuição do tempo entre a realização da ação e avaliação dos resultados nestas localidades; o número de domicílios trabalhados em termos percentuais não retrata a dimensão da área, principalmente quando se tem localidades em que foram trabalhados 19.260 domicílios na zona urbana a 26 por localidade na zona rural. O ano de 1998 foi um ano atípico, no qual, o número de casos humanos registrados e a prevalência canina foram os mais baixos do período estudado, podendo ser atribuído à subnotificação dos casos, desestruturação do serviço de atendimento dos casos humanos, diminuição das ações de controle por deficiência de recursos humanos e materiais; as variáveis mantêm interdependência, ou seja, as ações sofrem influência umas das outras; o longo período decorrido entre a coleta e a eliminação dos cães soropositivos, em torno de 40 a 80 dias, além da reposição imediata realizada pela população após a eliminação dos cães. Segundo Dye (1996), os novos cães tornam-se infectados em aproximadamente dois meses.

Os problemas relativos ao controle de agravos e suas perspectivas remetem à necessidade da realização de estudos e levantamentos, tais como inquéritos soro-epidemiológicos humanos, pois indivíduos expostos poderão se infectar e se recuperar sem conseqüências de morbidade, podendo funcionar como reservatório potencial, necessitando assim serem incluídos como dados epidemiológicos. Segundo Badaró et al. (1986), para cada caso clínico de LV há pelo menos 18,5 portadores assintomáticos ou subclínicos e em áreas de alta prevalência, para cada 6,5 portadores assintomáticos tem-se um doente. No Ceará esta relação foi de um doente para cada 11 assintomáticos.

Em estudo realizado com crianças na faixa etária de 0 a 5 anos no Município de Raposo, Maranhão, a relação infecção doença foi de 119:1 segundo o teste de IDRM e 28:1 de acordo com ELISA (Caldas et al., 2001).

Outros reservatórios silvestres devem ser investigados, visando avaliar a real incidência ou prevalência da doença, assim como definir o real padrão zoonótico ou antropozoonótico da doença no Brasil.

Para o diagnóstico do reservatório doméstico (cão) utilizou-se técnica de menor sensibilidade, quando comparada ao ELISA, sem contar no grande período que decorreu entre a coleta da amostra e a posterior eliminação do cão.

Quanto ao vetor, as pesquisas devem ser direcionadas ao mapeamento da fauna flebotomínica, associada às condições ambientais de cada região endêmica com o objetivo de conhecer a biologia do vetor no ambiente natural, para seu posterior controle, pois ações de borrifação são direcionadas à eliminação da população adulta do flebótomo. O inquérito entomológico foi realizado de forma descontinuada.

Como se trata de uma doença ligada às precárias condições sócio-econômicas e sanitárias, além das medidas de controle realizadas de forma sistematizada, faz-se necessário também definições de políticas públicas que garantam a resolução das distorções e desigualdades existentes nos padrões de saúde, ultrapassando limites das ações desse setor, onde novas alternativas deveriam ser incorporadas como acesso à educação, habitação, renda, suplementação alimentar, saneamento básico e 
ambiental que provavelmente teriam um novo impacto na ocorrência da doença.

O PCL está sendo reavaliado pelo MS, principalmente devido ao aumento do número de casos da doença, apesar da realização das ações de controle.

Os achados deste estudo apontam aspectos relevantes para a reflexão das ações de saúde que são propostas e que são implementadas.
Contudo, deve-se considerar que este estudo, baseado no uso de metodologia epidemiológica, deteve-se na quantificação de eventos. Como os aspectos envolvidos nas ações de saúde são na sua maioria de grande complexidade, podem não ser adequadamente investigados usando-se apenas técnicas de mensuração. Portanto, os achados produzidos devem ser observados com cautela.

\section{Agradecimentos}

Ao Prof. Dr. Edson Duarte Moreira Jr. (Fundação Oswaldo Cruz/Bahia) pelo incentivo e contribuição na elaboração deste estudo.

\section{Referências}

ASHFORD, D. A.; DAVID, J. R.; FREIRE, M.; DAVID, R.; SHERLOCK, I.; EULÁLIO, M. C.; SAMPAIO, D. P. \& BADARÓ, R., 1998. Studies on control of visceral leishmaniasis: Impact of dog control on canine and human visceral leishmaniasis in Jacobina, Bahia, Brazil. American Journal of Tropical Medicine, 55:53-57.

BADARÓ, R.; JONES, T. C.; LOURENÇO, R.; CERF, B. J.; PEDRAL-SAMPAIO, D.; CARVALHO, E. M.; ROCHA, H.; TEIXEIRA, H. \& JONHSON Jr., W. D. A., 1986. Prospective study of visceral leishmaniasis in an endemic area of Brazil. Journal of Infectious Diseases, 154:639-649.

CALDAS, A. J. M.; SILVA, D. R. C.; PEREIRA, C. C. R.; NUNES, P. M. S.; SILVA, B. P.; SILVA, A. A. M.; BARRAL, A. \& COSTA, J. M. L., 2001. Infecção por Leishmania (leishmania) chagasi em crianças de uma área endêmica de leishmaniose visceral americana na Ilha de São Luís - MA, Brasil. Revista da Sociedade Brasileira de Medicina Tropical, 34:15-21. 
COSTA, C. H. N.; PEREIRA, H. F. \& ARAÚJO, M. V., 1990. Epidemia de leishmaniose visceral no Estado do Piauí, Brasil, 1980-1986. Revista de Saúde Pública, 24:361-372.

DIETZE, R.; BARROS, G. B.; TEIXEIRA, L.; HARRIS, J.; MICHAELSON, K.; FALQUETO, A. \& COREY, R., 1997. Effect of eliminating seropositive canines on the transmission of visceral leishmaniasis in Brasil. Clinical Infectious Diseases, 25:1240-1242.

DYE, C., 1996. The logic of visceral leishmaniasis control. American Journal of Tropical Medicine and Hygiene, 55:125-130.

EVANS, T. G.; VASCONCELOS, I. A. B.; LIMA, J. W.; TEIXEIRA, J. M.; MACRULLIFE, I. T.; LOPES, U. G.; PEARSON, R. D. \& VASCONCELOS, A. W., 1990. Canine visceral leishmaniasis in northeast Brazil: Assessment of serodiagnosis methods. American Journal of Tropical Medicine and Hygiene, 42:118123.

GOVERNO DO ESTADO DA BAHIA, 1998. Diagnósticos de Municípios. Salvador: Secretaria da Indústria, Comércio e Mineração, Governo do Estado da Bahia.

IBGE (Fundação Instituto Brasileiro de Geografia e Estatística), 2000. Censo Demográfico e Estimativas. Junho $2002<\mathrm{http}$ ///www.ibge.org.br>.

HOSMER, D. W. \& LEMESHOW, S., 1989. Applied Logistic Regression. New York: John Wiley \& Sons.

LACERDA, M. M., 1994. The Brazilian leishmaniasis control program. Memórias do Instituto Oswaldo Cruz, 89:489-495.

MARZOCHI, M. C. A., 1985. Leishmaniose visceral canina no Rio de Janeiro - Brasil. Cadernos de Saúde Pública, 1:432-446.

MARZOCHI, K. B. F.; CALDERON, J. M. L. \& BONFIM, M. L., 1986. Calazar no Brasil: Problema em ascensão. ARS CURANDI - A Revista do Clínico Geral, 5:139-144.

MS (Ministério da Saúde), 1985. Relatório Anual da Campanha contra as Leishmanioses. Salvador: Diretoria Regional da Bahia, Superintendência de Campanhas de Saúde Pública, MS.

MS (Ministério da Saúde), 1996. Controle, Diagnóstico e Tratamento de Leishmaniose Visceral (Calazar) - Normas Técnicas. Brasília: Fundação Nacional de Saúde.
MS (Ministério da Saúde), 1999. Boletim Epidemiológico: Evolução Temporal das Doenças de Notificação Compulsória no Brasil de 1980 a 1998. Brasília: Centro Nacional de Epidemiologia, Fundação Nacional de Saúde, MS.

OLIVEIRA, S. S.; SILVA, S. S. B.; CORREIA, J. E. \& SANTOS, J. F., 1998. Leishmaniose visceral em Feira de Santana: inquérito sorológico em cães e a relação entre a distribuição da positividade canina por localidade e os casos humanos. Revista Brasileira de Análises Clínicas, 30:61-63.

PARANHOS-SILVA, M.; FREITAS, L. A. R.; SANTOS, W. C.; GRIMALDI Jr., G.; PONTES-DE-CARVALHO, L. C. \& OLIVEIRA-DOS-SANTOS, A. J., 1996. A crosssectional serodiagnostic survey of canine leishmaniasis due to Leishmania chagasi. American Journal of Tropical Medicine and Hygiene, 55:39-44.

PEREIRA, M. G., 1995. Epidemiologia: Teoria e Práti$c a$. Rio de Janeiro: Guanabara Koogan.

SESAB (Secretaria de Saúde do Estado da Bahia), 1999. Relatório. Salvador: Diretoria de Vigilância Epidemiológica, Superintendência de Vigilância e Proteção da Saúde, SESAB.

SENRA, M. S.; PIMENTEL, P. S. R. \& SOUZA, P. E. F. P., 1986. Leishmaniose visceral em Santarém, Pará. Aspectos gerais do controle. Inquérito Sorológico de cães e tratamento dos casos humanos. Hitéia Médica, Belém, 7:23-32.

SHERLOCK, I. A., 1996. Ecological interactions of visceral leishmaniasis in the State of Bahia, Brazil. Memórias do Instituto Oswaldo Cruz, 91:671-683.

SHERLOCK, I. A., 1997. Interações Ecológicas da Leishmaniose Visceral no Estado da Bahia, Brasil. Tese de Doutorado, Salvador: Fundação Oswaldo Cruz.

SHERLOCK, I. A.; MIRANDA, J. C.; SADIGURSKY, M. \& GRIMALDI Jr., G., 1984. Natural infection of the opossum Didelphis albiventris (Marsupialia Didelphidae) with Leishmania donovani in Brazil. Memórias do Instituto Oswaldo Cruz, 79:511.

SILVA, J. R., 1957. Leishmaniose Visceral (Calazar). Rio de Janeiro: Serviço Nacional de Educação Sanitária.

VIEIRA, J. B. F. \& COELHO, G. E., 1998. Leishmaniose visceral ou calazar: Aspectos epidemiológicos e de controle. Revista da Sociedade Brasileira de Medicina Tropical, 31:85-92.

Recebido em 5 de dezembro de 2002

Versão final reapresentada em 16 de abril de 2003

Aprovado em 22 de julho de 2003 